\title{
Quantum fields in anti-de Sitter wormholes
}

\author{
Carlos Barceló \\ Instituto de Astrofísica de Andalucía, CSIC, Camino Bajo de Huétor, 18080 Granada, Spain \\ and Instituto de Matemáticas y Física Fundamental, CSIC, C/Serrano 121, 28006 Madrid, Spain \\ Luis J. Garay \\ Instituto de Matemáticas y Física Fundamental, CSIC, C/Serrano 121, 28006 Madrid, Spain \\ (Received 16 July 1997; revised manuscript received 29 January 1998; published 27 March 1998)
}

\begin{abstract}
We study the effects of asymptotically anti-de Sitter wormholes in low-energy field theory and give a general prescription for obtaining the local effective interaction terms induced by them. The choice of vacuum for the matter fields selects a basis of the Hilbert space of anti-de Sitter wormholes whose elements can be interpreted as states containing a given number of particles. This interpretation is subject to the same kind of ambiguity in the definition of a particle as that arising from quantum field theory in curved spacetime. [S0556-2821(98)05210-2]
\end{abstract}

PACS number(s): 04.60.Ds, 04.62.+v, 98.80.Hw

Among the possible kinds of fluctuations that might appear in quantum gravity, wormholes describe processes that involve baby universes branching off and joining onto asymptotically large regions of spacetime. Wormholes have been extensively studied in the literature [1]. It has been claimed that, in the dilute wormhole approximation, their effects in low-energy fields can be taken into account through the introduction of local effective interactions [2,3]. As a consequence, they would modify the coupling constants of any low-energy effective physical theory. These effective interactions produced by wormholes have been calculated for a variety of matter fields coupled to gravity [4-9]. In all these calculations, the asymptotically large region of the wormhole has been assumed to be flat. Therefore, the effective interactions produced by such wormholes have been modeled by interacting terms added to a free quantum field theory in flat spacetime.

In a previous paper [10], we dealt with wormholes with other types of asymptotic behavior; in particular, we studied the case of wormholes in asymptotically anti-de Sitter (AdS) spacetimes. This exhausts all possible maximally symmetric asymptotic behaviors. Also, AdS wormholes could be regarded as excited states in the sense that the cosmological constant $\Lambda$ could be interpreted as a non-vanishing asymptotic energy of the matter field. We found an appropriate action for these wormholes and showed that they give a non-vanishing contribution to the path integral.

The calculation of the possible local effective interactions produced by $\mathrm{AdS}$ wormholes is of particular interest. We will see that wormholes in AdS spacetime do not contribute to the cosmological constant nor to the gravitational coupling constant directly. However, the presence of a cosmological constant will certainly modify the form of the effective interactions produced by wormholes, in comparison with the flat case, when these are not spherically symmetric. Another interesting characteristic of this model is that there naturally exist different possible vacuum choices for the matter fields in the asymptotic regions, as happens in quantum field theory in curved spacetime. There is a relation between the definition of this vacuum and the choice of basis in the Hilbert space of quantum wormholes that allows us to interpret the basis elements as states of wormholes containing a certain number of particles.

The aim of this work is to model the effects of closed universes that branch off or join onto an asymptotically AdS spacetime by means of an effective quantum field theory in an AdS background. We will consider processes that involve a single baby universe. In the dilute wormhole approximation, the global effects will be the result of these basic processes [2,11]. In order to find these effects on the matter fields, and following previous analyses in flat backgrounds [4-9], we will calculate matrix elements of products of operators $\Phi(x)$, each representing a generic matter field at a different point, between a specific vacuum $|0\rangle$, and the elements $\left|\Psi_{\alpha}\right\rangle$ of a basis of the Hilbert space of AdS wormholes [4]:

$$
\left\langle 0\left|\Phi\left(x_{1}\right) \cdots \Phi\left(x_{r}\right)\right| \Psi_{\alpha}\right\rangle \text {. }
$$

In flat spacetime, the state that is void of particles for inertial observers defines a preferred vacuum for which its associated propagator is asymptotically damped. In AdS spacetime, we can define a one-parameter family of maximally symmetric vacua by analogy with the de Sitter case [12] (although there are some subtleties in the definition of states in an AdS Lorentzian background because this spacetime does not possess a well-posed Cauchy problem, we will work in the Euclidean sector where this issue does not arise [13]). The propagator associated with each vacuum of this family is damped when the geodesic distance between the two points becomes large, but with a different fall-off for each vacuum choice [14]. This family of vacua is the analogue in AdS spacetime of the vacua defined by uniformly accelerated reference frames in the flat case. The state $|0\rangle$ is any fixed vacuum of this family. 
The wormhole states $|\Psi\rangle$ will be represented by wave functions $\Psi\left[g_{i j}^{\prime}, \Phi^{\prime}\right]$ that are solutions of the WheelerdeWitt equation and satisfy the wormhole boundary conditions [15]. The arguments of these wave functions are the three-geometry and the matter content of the system under consideration. We will restrict our study to spacetime geometries that can be foliated in nearly spherically symmetric three-surfaces, which will be described by a scale factor and small perturbations around the homogeneous configuration, and bosonic massless fields as matter content. In an appropriate gauge, we can just keep as configuration variables the scale factor and the different coefficients that represent the true degrees of freedom of the matter fields in an expansion in terms of hyperspherical harmonics on the three-sphere $[16,17]$. For conformally coupled matter fields, such as a conformal scalar field or an electromagnetic field, the scale factor decouples from the matter degrees of freedom in the Wheeler-deWitt equation. The part of the wave function that contains the scale factor is asymptotically damped and indicates the existence of an asymptotically AdS region. On the other hand, each mode $n$ in the harmonic expansion of the matter content can be described as a quantum harmonic oscillator with a frequency that only depends on the specific mode $n$. As we will see, each quantum of energy of these oscillators associated with the mode $n$ can be interpreted as a particle of mode $n$ in the conformal vacuum, that is, in the vacuum conformally related to the natural vacuum in flat spacetime.

When the matter field is coupled in a non-conformal way to gravity, as happens with gravitons [18], we cannot carry out the above separation of geometrical and matter degrees of freedom. The different matter harmonic oscillators would have frequencies that depend on the scale factor and therefore we could not interpret them as particles. The creation of particles generated by the expansion of these systems does not allow us to interpret the different wormhole states as containing certain number of particles.

Finally, an important restriction on wormhole states is due to the linearization instability of the perturbations to the rotationally invariant configurations $[7,19]$. In other words, the invariance under the isotropy group $S O(4)$ restricts the Hilbert space of wormhole states to those that contain only rotationally invariant matter states. This means that, for the inhomogeneous modes, single-particle wormhole states are not allowed.

We are interested in processes in which there is creation or annihilation of particles associated with a specific vacuum in two different points of a spacetime region where a wormhole end is inserted. The matrix element $\left\langle 0\left|\Phi\left(x_{1}\right) \Phi\left(x_{2}\right)\right| \Psi_{\alpha}\right\rangle$ is then given, following Hawking [4], by a path integral over geometries and matter fields that induce a metric $g_{i j}^{\prime}$ and a value $\Phi^{\prime}$ for the matter field in a cross section of the wormhole, and that satisfy some asymptotic requirements that guarantee the asymptotically AdS behavior and that select a vacuum for the matter field in the semiclassical approximation. Then, we will integrate over all possible configurations $\left(g_{i j}^{\prime}, \Phi^{\prime}\right)$ weighted with the wormhole wave function. Thus, the matrix element has the form

$$
\begin{aligned}
\left\langle 0\left|\Phi\left(x_{1}\right) \Phi\left(x_{2}\right)\right| \Psi_{\alpha}\right\rangle & \\
= & \int \mathcal{D} \Phi^{\prime} \mathcal{D} g_{i j}^{\prime} \Psi_{\alpha}\left[g_{i j}^{\prime}, \Phi^{\prime}\right] \\
& \times \int \mathcal{D} \Phi \mathcal{D} g_{\mu \nu} \Phi\left(x_{1}\right) \Phi\left(x_{2}\right) e^{-I\left[g_{\mu \nu}, \Phi\right]},
\end{aligned}
$$

plus the appropriate gauge fixing condition and FadeevPopov determinant. We will evaluate this path integral semiclassically. The action $I\left[g_{\mu \nu}, \Phi\right]$ contains a surface term that renders it finite for classical solutions [10], a necessary requirement for the semiclassical approximation to be meaningful. The saddle point solution for the gravitational part must be an asymptotically AdS spacetime. As far as the lowenergy regime is concerned, it can be taken to be pure AdS spacetime outside a three-sphere in which the wave function takes its arguments.

The position of the spacetime points $x_{1}, x_{2}$ can only be specified modulo the isometries of Euclidean AdS spacetime, $S O(4,1)$. This group is isomorphic to the semi-direct product of AdS translations and the isotropy group $S O(4)$. If $\Phi(x)$ is a saddle point solution for the matter field, then $\Phi_{x^{\prime} h}(x)$, the transform of $\Phi(x)$ under the isometry $\left(x^{\prime} h\right)^{-1}$, is also a solution, $x^{\prime}$ being an element of the group of translations in AdS spacetime and $h \in S O(4)$. As a consequence, one has to average over $S O(4,1)$. The invariant Haar measure in this group is equivalent to integrating first over the group $S O(4)$ and, then, over the coset space, i.e. over AdS spacetime [20]. We can interpret this integral as an average over the orientations $h$ of the wormhole and the positions $x^{\prime}$ in which it can be inserted. In flat spacetime, similar results can be obtained, if we use its own group of isometries, the Euclidean group in four dimensions $E_{4}$ [4].

In an appropriate gauge, we can expand the massless bosonic field under consideration in terms of hyperspherical harmonics in a way that only the true matter field degrees of freedom are present. Let

$$
\Phi_{x^{\prime} h}(x)=\sum_{n, \sigma_{n}} c_{n}\left[\mu\left(x, x^{\prime}\right)\right] M_{h}^{n, \sigma_{n}} \mathcal{H}_{n, \sigma_{n}}\left(\widehat{x-x^{\prime}}\right)
$$

be such an expansion, where $n$ is the mode of the hyperspherical harmonic $\mathcal{H}_{n, \sigma_{n}}(\widehat{x-x})$, the hat denotes unit vectors, $c_{n}\left[\mu\left(x, x^{\prime}\right)\right]$ are functions of the geodesic distance $\mu\left(x, x^{\prime}\right)$ between $x$ and $x^{\prime}$ and $\sigma_{n}$ is an index that covers the degeneration space of the harmonic mode $n$. This space carries an irreducible representation of the group $S O(4)$ [20]. If we use a tensor form for this representation, $M_{h}^{n, \sigma_{n}}$ will denote the components $\sigma_{n}$ of a tensor that belongs to the representation associated with the mode $n$ and rotated by an element $h$ of $S O(4)$. Taking into account that these irreducible tensor representations of $S O(4)$ are characterized by the different kinds of symmetries under permutations of their tensor indices $\sigma_{n}[16]$ and that the propagator of the matter field in AdS spacetime $G\left(x, x^{\prime}\right)$ and the saddle point $\Phi_{x^{\prime} h}(x)$ satisfy the same equation, we can easily conclude that this saddle point can be expressed for each harmonic mode $n$ in terms of the propagator $G$ alone. Explicitly, $\Phi_{x^{\prime} h}^{n}(x)=\mathcal{M}_{h}^{n} \cdot \Theta_{n}^{\prime} G\left(x, x^{\prime}\right)$, where now $\mathcal{M}_{h}^{n}$ is a constant 
tensor that contains all the dependence on the arguments of the wave function and the rotation group, and the dot represents a contraction of all primed tensor indices. The operator $\Theta_{n}$ can be constructed recursively by symmetrizing $\nabla \Theta_{n-1}$ and substracting all its traces. The operator $\Theta_{n_{0}}$ for the lowest mode is given by the smallest irreducible representation of each matter field and is therefore specific to each matter content: it is the identity for the scalar field, the antisymmetric derivative in the electromagnetic case and, for gravitons, it has the same derivative structure as the Weyl tensor $C^{\mu \nu \rho \sigma}$. Furthermore, for fields with spin, like photons and gravitons, we have to deal with both helicities, positive and negative, separately. This amounts to introducing operators $\Theta_{n \pm}$ which are the self-dual and anti-self-dual parts of $\Theta_{n}$, i.e. $\Theta_{n \pm}=\Theta_{n} \pm * \Theta_{n}$, the asterisk denoting the Hodge dual. For fermions or massive fields, the representation theory provides analogous results, although the explicit expressions are slightly more involved.

In view of the simple form of the saddle points, it is easily seen that the wormhole matrix element contains the product $\left[\mathcal{M}_{h}^{n} \cdot \Theta_{n}^{\prime} G\left(x_{1}, x^{\prime}\right)\right]\left[\mathcal{M}_{h}^{n} \cdot \Theta_{n}^{\prime} G\left(x_{2}, x^{\prime}\right)\right]$. Using the property [20] that, for any irreducible representation $R(h)$ of the group $S O(4), \quad \int_{S O(4)} d h R(h) \otimes R(h)=1 \otimes 1$, the average over rotations yields

$$
\left(\mathcal{M}^{n} \cdot \mathcal{M}^{n}\right)\left[\Theta_{n}^{\prime} G\left(x_{1}, x^{\prime}\right) \cdot \Theta_{n}^{\prime} G\left(x_{2}, x^{\prime}\right)\right]
$$

and the matrix element becomes

$$
\begin{aligned}
& \left\langle 0\left|\Phi\left(x_{1}\right) \Phi\left(x_{2}\right)\right| \Psi_{\alpha}\right\rangle \\
& \quad=K\left(0, \Psi_{\alpha}\right) \int d^{4} x^{\prime} \sqrt{g\left(x^{\prime}\right)} \Theta_{n}^{\prime} G\left(x_{1}, x^{\prime}\right) \cdot \Theta_{n}^{\prime} G\left(x_{2}, x^{\prime}\right),
\end{aligned}
$$

where $K\left(0, \Psi_{\alpha}\right)$ is a constant that depends on the choice of vacuum and on the state $\left|\Psi_{\alpha}\right\rangle$.

For conformal matter fields and choosing the conformal vacuum, the path integral that appears in the definition of this two-point matrix element gives separate contributions for the scale factor and the matter harmonic modes. The only wave function that gives a nonvanishing result for $K\left(0, \Psi_{\alpha}\right)$ is that which contains only two quanta in the mode $n$, apart from the vacuum itself $[4,6]$. A similar analysis, applied to matrix elements of more than two points, allows us to interpret each state of the basis of the Hilbert space of wormholes characterized by the number of energy quanta of the matter harmonic oscillator in the mode $n$, as a quantum wormhole that contains a given number of particles in that mode associated with the conformal vacuum. For other vacuum choices, the path integral does not separate but still, analogous interpretations for different bases of the wormhole Hilbert space can be reached. Therefore, for each vacuum choice, there exists an orthonormal basis in the Hilbert space of wormholes such that its elements can be labeled by the number of particles that they contain. Thus, the ambiguity in the choice of vacuum which is present in quantum field theory in curved spacetimes also shows up in wormhole physics. When the matter content is not conformally coupled, as in the graviton case, we cannot label the wormhole states by their particle content because there exists creation of particles owing to the expansion of the asymptotic region. The states are global states particle-background.

Let us now find an interaction Lagrangian that, via the formula

$$
\left\langle 0\left|\Phi\left(x_{1}\right) \Phi\left(x_{2}\right) \int d^{4} x^{\prime} \sqrt{g\left(x^{\prime}\right)} \mathcal{L}_{I}\left[\Phi\left(x^{\prime}\right)\right]\right| 0\right\rangle,
$$

where, here, $|0\rangle$ represents a matter field vacuum in AdS background, reproduces the matrix element above, up to a constant factor. It is straightforward to see that the required interaction Lagrangian is

$$
\mathcal{L}_{I}=(\Theta \Phi)^{2} .
$$

Indeed, the linearity of the operator $\Theta$ and Wick's theorem allow us to write the two-point function above in the form

$$
\int d^{4} x^{\prime} \sqrt{g\left(x^{\prime}\right)} \Theta^{\prime} G\left(x_{1}, x^{\prime}\right) \cdot \Theta^{\prime} G\left(x_{2}, x^{\prime}\right) .
$$

Let us now apply these results to three separate kinds of matter fields, namely, a conformal scalar field, and an electromagnetic field, which are conformally coupled to gravity, and gravitons, which are not because of the presence of a cosmological constant. For the lowest mode, this Lagrangian reads $\mathcal{L}_{I}=\phi^{2}$ for the scalar field, $\mathcal{L}_{I}=F_{\mu \nu} F^{\mu \nu}$ for photons and $\mathcal{L}_{I}=C_{\mu \nu \rho \sigma} C^{\mu \nu \rho \sigma}$ for gravitons, which coincide with the results obtained for asymptotically flat wormholes [4-7]. In the case of photons and gravitons, positive and negative helicities provide the same interaction Lagrangian, as expected, because the cross product $\Theta_{n} \Phi \cdot{ }^{*} \Theta_{n} \Phi$ is a topological invariant, as can be checked by direct calculation. If we call $n_{0}$ to this lowest mode for each matter content $\left(n_{0}=1,2,3\right.$ for scalars, photons and gravitons, respectively), the operator $\Theta_{n_{0}+1}$ for the next mode is, following the prescription described above, $\nabla \Theta_{n_{0}}$, where the required symmetrization is automatically implemented. Therefore, the effective interaction Lagrangian for this mode $n_{0}+1$, as well as that for the lowest one $n_{0}$, does not depend on the cosmological constant and has the same form as that obtained in flat spacetime. For the next higher mode, $\Theta_{n_{0}+2}$ contains a symmetrized and traceless combination of products of two derivatives. In particular, it will contain a term of the form $g^{\mu \nu} \nabla^{2} \Theta_{n_{0}}$, which is proportional to the cosmological constant by virtue of the equation for the propagator in AdS spacetime. For instance, the effective interaction Lagrangian coming from the $n=3$ inhomogeneous mode of a scalar field is of the form $\left(\nabla^{\mu} \nabla^{\nu} \phi-\frac{1}{6} \Lambda g^{\mu \nu} \phi\right)^{2}$. We then see that the interaction Lagrangians for higher modes in AdS spacetime and those in the flat case differ in terms that are proportional to powers of the cosmological constant. It is also worth noting that $\mathrm{AdS}$ wormholes do not induce any direct modification to the cosmological term nor to Newton's constant.

Let us conclude with a brief summary. We have given a general prescription for finding the low-energy effective interaction Lagrangians induced by wormholes in a maximally symmetric asymptotically large region of spacetime. We have seen that the two lowest modes in the harmonic expansion of each matter field give interaction Lagrangians of the 
same form as those obtained in asymptotically flat spacetimes, while the higher inhomogeneous modes give Lagrangians that contain an explicit dependence on the cosmological constant.

We are very grateful to G.A. Mena Marugán, M. Moles and P.F. González-Díaz for helpful discussions and suggestions. C.B. was supported by a Spanish Ministry of Educa- tion and Culture (MEC) grant. C.B. is also grateful to J.B. Hartle and the Institute for Theoretical Physics (UCSB), where part of this work was done, for warm hospitality. This research was supported in part by the National Science Foundation under Grant No. PHY994-07194. L.J.G. was supported by funds provided by DGICYT and MEC (Spain) under Contract Adjunct to Project No. PB94-0107.
[1] A. Strominger, in Particles, Strings and Supernovae, edited by A. Jevicki and C.I. Tan (World Scientific, Singapore, 1989); S.W. Hawking, Mod. Phys. Lett. A 5, 145 (1990); 5, 453 (1990); L.J. Garay, Ph.D. thesis, Universidad Autónoma de Madrid, 1992, and references therein.

[2] S. Coleman, Nucl. Phys. B307, 867 (1988).

[3] S.W. Hawking, Nucl. Phys. B335, 155 (1990).

[4] S.W. Hawking, Phys. Rev. D 37, 904 (1988).

[5] A. Lyons, Nucl. Phys. B324, 253 (1989).

[6] H.F. Dowker, Nucl. Phys. B331, 194 (1990).

[7] H.F. Dowker and R. Laflamme, Nucl. Phys. B366, 209 (1991).

[8] L.J. Garay and J. García-Bellido, Nucl. Phys. B400, 416 (1993).

[9] A. Lukas, Nucl. Phys. B442, 533 (1995).

[10] C. Barceló, L.J. Garay, P.F. González-Díaz, and G.A. Mena Marugán, Phys. Rev. D 53, 3162 (1996).

[11] L. Klebanov, L. Susskind, and T. Banks, Nucl. Phys. B317, 665 (1989).
[12] C.J.C. Burges, Nucl. Phys. B247, 533 (1984); B. Allen, Phys. Rev. D 32, 3136 (1985).

[13] S.J. Avis, C.J. Isham, and D. Storey, Phys. Rev. D 18, 3565 (1978).

[14] B. Allen and T. Jacobson, Commun. Math. Phys. 103, 669 (1986).

[15] S.W. Hawking and D.N. Page, Phys. Rev. D 42, 2655 (1990).

[16] E.M. Lifshitz, J. Phys. (USSR) 10, 116 (1946); E.M. Lifshitz and I. Khalatnikov, Adv. Phys. 12, 185 (1963).

[17] J.J. Halliwell and S.W. Hawking, Phys. Rev. D 31, 1777 (1985).

[18] L.P. Grishchuk, Zh. Eksp. Teor. Fiz. 67, 825 (1974) [Sov. Phys. JETP 40, 409 (1975)]; S. Wada, Nucl. Phys. B276, 729 (1986).

[19] V. Moncrief, Phys. Rev. D 18, 983 (1978).

[20] A. Barut and R. Raczka, Theory of Group Representations and Applications (World Scientific, Singapore, 1986). 